\title{
PACING STRATEGY OF THE FINISHERS OF THE WORLD MARATHON MAJORS SERIES
}

\author{
Ülari Kais, Kerli Mooses, Rasmus Pind, Ando Pehme, Priit Kaasik, and Martin Mooses \\ Institute of Sport Sciences and Physiotherapy, University of Tartu, Tartu, Estonia
}

Original scientific paper

https://doi.org/10.26582/k.51.1.5

\begin{abstract}
:
The purpose of the study was to describe pacing patterns of the finishers of the World Marathon Majors series and the effect of sex and age on the pacing pattern. The finishers of the World Marathon Majors series, a total of 69814 male runners and 46856 female runners with finishing time $\leq 6$ hours were included in the analysis. Difference in pacing (dev\%) was calculated as a difference between the first and second half of the marathon and expressed as a percentage of time. Analysis of variance (ANOVA) was used to evaluate the differences within and between the marathon time groups. The differences between the first and second half of the marathon by sex and age group were analysed using linear regression. The average difference between the first and second half of the marathon was $3.44 \pm 2.67 \%$ for male and $2.81 \pm 2.10 \%$ for female runners. Male runners with finishing times of 3:00 (h:min) and females with 4:00 (h:min) or slower had the significantly faster first half of the marathon compared to the evenly paced marathon ( $\mathrm{p}=.038$ and $\mathrm{p}=.001$, respectively). Regression analysis revealed that female runners had $0.26 \%$ smaller difference between the first and second half of the race compared to male runners $\left(\mathrm{R}^{2}=0.256 ; \mathrm{p}<.001\right)$ when controlled for age and time group. Also, veterans (40 years and older) paced more evenly compared to non-veterans $\left(\mathrm{R}^{2}=0.256\right.$; $\mathrm{p}<.001)$. In conclusion, irrespective of sex and age, faster finishers maintain a more constant velocity than the slower ones. In addition, women and veterans present more even pacing strategy compared to men and non-veterans, respectively.
\end{abstract}

Key words: distance running, recreational runners, pacing

\section{Introduction}

During the last 45 years, there has been a marked increase in the number of marathon races that occur annually - from 644 races in 1990 to 2820 races in 2011 - as well as in the number of participants - 234000 registered in 1990 and 1.48 million in 2011 (ARRS, 2016). Six of the world most renowned marathons belong to The Abbott World marathon series: Tokyo, Boston, London, Berlin, Chicago and New York City Marathon (AWMM, 2016). The marathon is an endurance event where choosing the best pacing strategy can be crucial in achieving the personal best performance (Hanley, 2016). Thus, in the light of a growing number of participants with very different training background in marathon running events, the optimal pacing deserves closer attention.

Pacing is the actual distribution of speed and power output or energetic reserves during a given sporting event (Roelands, de Koning, Foster, Hettinga, \& Meeusen, 2013) and is a fundamental determinant factor in competitive endurance perfor- mance (Foster, et al., 2004). Pacing strategy is a self-selected strategy or tactic that athletes adopt from the beginning of an event (Roelands, et al., 2013). More specifically, the intriguing aspect of investigating the variability of pace is that the runner, theoretically, selects a pace to maintain his/ her homeostasis (Haney \& Mercer, 2011). However, if the wrong pace is selected, then fatigue will occur and the runner will slow down or possibly drop out from the race (Haney \& Mercer, 2011). Numerous studies (Abbiss \& Laursen, 2008; Angus, 2014; Hanley, 2016; March, Vanderburgh, Titlebaum, \& Hoops, 2011) have recognized that appropriate pacing strategy can have a significant impact on athletes' performance. However, the mentioned studies have analysed only elite-level athletes and/ or have been conducted on a small sample size. By applying mathematical modelling to athletic performances, coaches and researchers have examined six different pacing strategies (Abbiss \& Laursen, 2008) from which the „even pacing” or "constant pace" is believed to be optimal for 
prolonged (longer than $2 \mathrm{~min}$ ) events (de Koning, Bobbert, \& Foster, 1999). There is a consensus that even pacing is best for optimizing performance in races that take several hours to complete (Abbiss \& Laursen, 2008), such as the marathon. To date, marathon pacing strategies for elite athletes are well studied (Ely, Martin, Cheuvront, \& Montain, 2008), however the majority of marathon participants are non-elite runners (Haney \& Mercer, 2011). Thus, little information is available in regard with pacing patterns displayed by recreational runners (Foster, et al., 2012; Roelands, et al., 2013). Moreover, to our knowledge only few previous studies have investigated sex differences in marathon pacing reporting a decrease in the speed independently of sex (Santos-Concejero, et al., 2014) and women tending to adopt more even pace compared to men (Deaner, Carter, Joyner, \& Hunter, 2015). This might be due to a lower respiratory exchange ratio during endurance exercise, which in turn, suggests glycogen-sparing effect to delay onset of glycogen depletion (March, et al., 2011). Additionally, a smaller variation in consecutive marathon times in older runners (50 years and older) compared to younger ones has been shown (Hopkins \& Hewson, 2001; March, et al., 2011). This difference could be explained by more competitive experience, attitudes towards competing and their experience of pacing from previous races (Hopkins \& Hewson, 2001; March, et al., 2011).

Surprisingly, there is a lack of available information on pacing over the $42.195 \mathrm{~km}$ distance especially given the popularity of the marathon running. Understanding the variability of pacing may lead to a better understanding of factors that influence marathon performance and help runners to either complete a marathon successfully or improve their race performance. Therefore, the aim of the present study is to describe the pacing patterns of the finishers of the World Marathon Majors series and the effect of sex and age on the pacing strategy based on the publicly available data from the race organisers.

\section{Methods}

\section{Participants}

The 2015 Abbot World Marathon Majors series included London, Chicago, New York, Boston, Tokyo and Berlin marathons. In the current analysis, publicly available data of the London $(n=36$ 434), Chicago $(n=34658)$ and New York $(n=45$ 578) marathon finishers with the finishing time $\leq$ $6 \mathrm{~h}$ were included. Boston marathon was excluded because the course was not eligible for the international record settings requirements due to not meeting IAAF Competition Rules (IAAF Competition Rules, 2014): (i) the start and finish points of a course, measured along a theoretical straight line between them, shall not be further apart than $50 \%$ of the race distance; (ii) the decrease in elevation between the start and finish shall not exceed an average of one in a thousand, i.e., $1 \mathrm{~m}$ per $\mathrm{km}$. Finish protocols of the Tokyo and Berlin marathons were publicly available on the race organisers' webpage; however, the data format was not suitable for further analyses. Thus the organisers of the Tokyo and Berlin marathons were contacted via e-mail to provide databases of the finish protocols (sex, age, half-marathon time and finishing time), but it was turned down referring to the protection of personal information of the participants.

Finish protocols of the London, Chicago and New York marathons included the following: sex, age group (18-39 years and $\geq 40$ years), half-marathon time and total time of finishers. For several runners with the finishing time $>6 \mathrm{~h}$ the half marathon time was missing in the protocol and therefore they were excluded from the further analysis. Race organisers categorized participants as (i) 18-39 years of age (non-veteran) or (ii) 40 years and older (veteran). For further analyses, all the finishers were categorized to the $15 \mathrm{~min}$ interval timegroups (Figure 1). The study was conformed to the Declaration of Helsinki.

Difference in pacing (dev\%) was calculated as a difference between the first and second half of the marathon and expressed as \% of time as follows:

$$
\operatorname{dev} \%=50-\left(\frac{T 2}{T} \times 100\right)
$$

where T2 is time of the second half of the marathon in seconds, $\mathrm{T}$ is the total time of the marathon in seconds. Negative value indicates the relatively faster first half of the marathon compared to the even pacing.

Track profile between the marathon races was considered by comparing pacing (duration of the first half of the marathon in relation to the second half) for the fastest 25 finishers from all the three marathons. There was no difference between the first and second half of the marathon for the first 25 finishers between the marathons ( $p=.751$ ), thus, potentially indicating a similar marathon track profile. Additionally, for the London, Chicago and New York marathon the lowest elevation was 0, 176, $0 \mathrm{~m}$ and the highest elevation was 55, 184 and $50 \mathrm{~m}$ above sea level, respectively. Ambient temperature at the time of the start for the London, Chicago and New York marathon was $9,12,15^{\circ} \mathrm{C}$ and relative humidity $81,70,62 \%$, respectively.

\section{Statistical methods}

All calculations were performed using the IBM SPSS Statistics v.20 (SPSS Inc, Chicago, IL, USA) software. Normality of all the variables was tested using Shapiro Wilk's test. Analysis of vari- 
Table 1. Participants of the study

\begin{tabular}{lccccc}
\hline & N & Men & Women & Veterans & Non-veterans \\
\hline Chicago & 34658 & 19150 & 15508 & 14403 & 20255 \\
London & 36434 & 23430 & 13004 & 16493 & 19941 \\
New York & 45578 & 27234 & 18344 & 24163 & 21415 \\
\hline Total & 116670 & 69814 & 46856 & 55059 & 61611 \\
\hline
\end{tabular}

ance (ANOVA) was used to evaluate the differences within and between the marathon time groups. In case of a significant main effect, Tukey's HSD posthoc analysis was used. Partial eta square $\left(\eta^{2}\right)$ was calculated. Effect sizes, as estimated through $\eta^{2} p$, were categorized as small (0.01), medium (0.06) or large (0.14) (Cohen, 1988). The differences between the first and second half of the marathon were analysed using linear regression and controlled for sex, age group and time group. The level of significance was set at $p<.05$.

\section{Results}

In total 116670 finishers from the London, Chicago and New York marathons were included in the study. There were more male finishers compared to female as well as more non-veteran compared to veteran finishers (Table 1).

The highest number of finishers among men was in time category of 3:45-4:00 (h:min) and in 4:30-4:45 (h:min) category for women. Distribution of the participants in 15 min time categories are shown in Figure 1.

There was a significant difference in the pacing between time groups $\left(\mathrm{F}_{1,15}=1858.35, p<.001\right.$, $\left.\eta^{2} \mathrm{p}=0.193\right)$. Male runners with finishing times 3:00 (h:min) and females with 4:00 (h:min) or slower had the significantly faster first half of the marathon

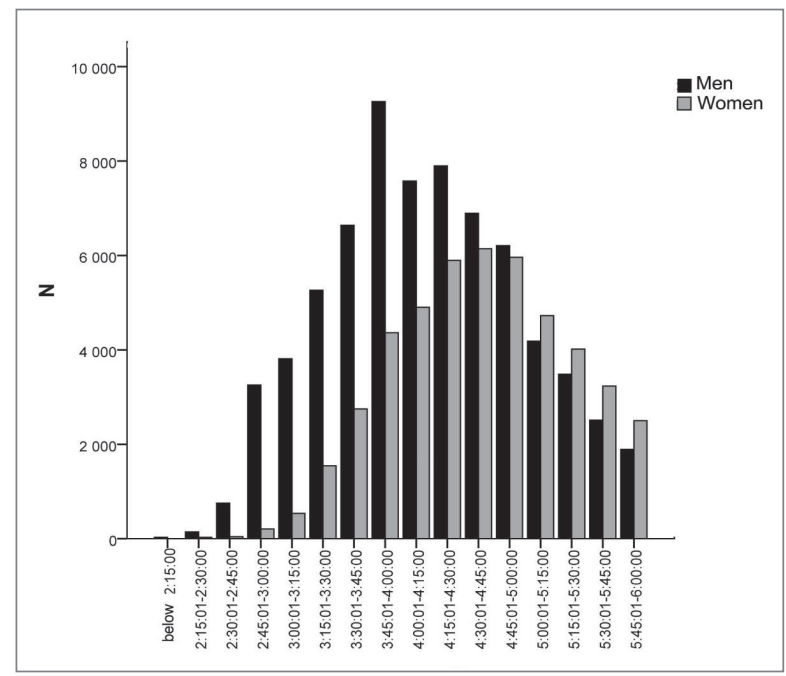

Figure 1. Distribution of the participants in 15 min time categories. compared to evenly paced marathon ( $p=.038$ and $p=.001$, respectively) (Figure 2). It is important to note the female runners' trend towards a significantly faster start compared to the second part of the race ( $p=.051)$ in the time group of 3:45-4:00 (h:min).

The average difference between the first and second half of the marathon was $3.44 \pm 2.67 \%$ for male and $2.81 \pm 2.10 \%$ for female runners, indicating a faster pace for the first half of the marathon. Men and women in the slower finish time groups had a more pronounced decline in the running speed during the second half of the race (Figure 2). When comparing male and female runners in the same time group, the decline in the pace for men was significantly more pronounced compared to female runners in the time group 2:15-2:30 (h:min) and in all time groups from 3:00$3: 15$ until the 6:00 (h:min) $\left(\mathrm{F}_{1,15}=54.49, p<.001\right.$, $\eta^{2} \mathrm{p}=0.007$ ). Regression analysis revealed that in the case of the same age and time group, female runners had by $0.26 \%$ smaller difference between the first and second half of the race compared to male runners $\left(\mathrm{R}^{2}=0.256 ; p<.001\right)$. Veterans had by $0.05 \%$ smaller difference between the first and second half of the marathon compared to nonveteran runners when controlled for time group and $\operatorname{sex}\left(\mathrm{R}^{2}=0.256 ; p<.001\right)$ (Figure 3$)$.

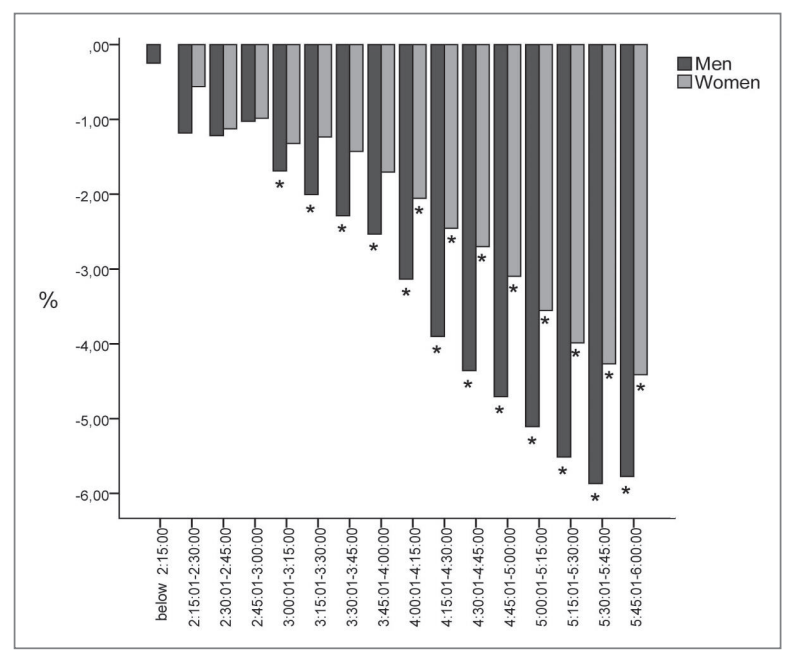

Figure 2. Differences between the first and second half of the marathon in different timegroups. * significantly different from the group of the fastest finishers of the same gender $(p<.05)$. 


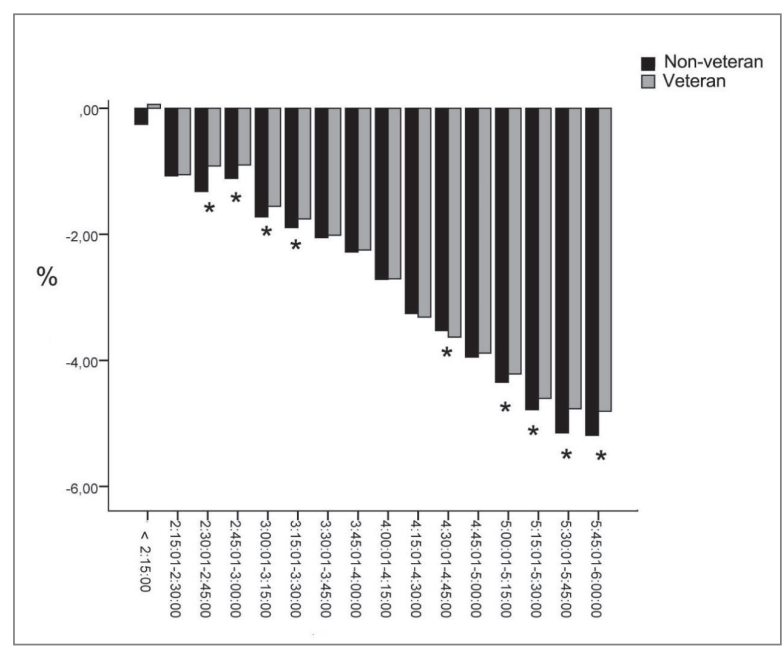

Figure 3. Differences in pace by finishing timegroup differenciated by veterans and non-veterans. * significantly different from veteran of the same group $(p<.05)$.

\section{Discussion and conclusions}

The aim of the present study was to compare the pacing profiles of marathon finishers between the first and second half of the running marathon races which belonged to the 2015 World Marathon Majors series. The main finding was that faster runners had smaller differences between the first and second half of the marathon compared to slower runners over the range of athletes with different performance level and that sex and age were important factors affecting the pacing strategy.

Santos-Lozano et al. (2014) discussed that both even pace and varied pacing patterns have been positively associated with marathon performance. Previous studies have proposed that the adoption of a positive pacing strategy results in an increased $\mathrm{VO}_{2}$ (Sandals, Wood, Draper, \& James, 2006; Thompson, MacLaren, Lees, \& Atkinson, 2003), greater accumulation of fatigue-related metabolites (Thompson, MacLaren, Lees, \& Atkinson, 2004; Thompson, et al., 2003) as well as in an increase in the rating of perceived exertion (Thompson, et al., 2004) during the early stages of an exercise task. As a result, a reduction in the exercise intensity and the observed positive pacing profile likely evolves in response to these signals to avoid catastrophic failure (Marino, 2004; Thompson, et al., 2004). On the other hand, in ultra-endurance events ( $>4$ hours), positive pacing strategy has been noted. According to a review by Abbiss and Laursen (2008), a reduction in intensity is potentially the result of increased glycogen depletion, which leads to an altered substrate utilization, neuromuscular fatigue and/or psychological factors associated with the individual perception of fatigue.

Those runners whose finishing time was $>3: 00$ (h:min) for male and > 4:00 (h:min) for female started the race using a relatively faster pace compared to the faster finishers or, on the other hand, they slowed down their pace more compared to the faster finishers. Present findings are somewhat in line with earlier studies (Ely, et al., 2008; Santos-Lozano, et al., 2014) where faster finishers ran at an even pace over the marathon distance. However, slower runners started out relatively faster than their average pace for the first $5 \mathrm{~km}$ before settling at a pace that they could maintain for 20 $\mathrm{km}$ or $25 \mathrm{~km}$, but after that, the pace of the slower finishers declined to a greater extent compared to the faster finishers (Ely, et al., 2008). It can be concluded that the faster finishers maintain a more consistent race pace throughout the race than the slower runners (Ely, et al., 2008; March, et al., 2011). Limitation of both mentioned studies is that they include a small sample size and the fact that in the former study (Ely, et al., 2008) only female athletes were included. Santos-Lozano et al. (2014) had a remarkable sample size, however they divided finishers into the four quartiles, compared to the 15 min time categories in the present study that enables better overview of the pacing strategy of finishers with more different abilities.

However, March et al. (2011) showed that age, sex and finish time were each statistically significant factors to influence marathon pacing. Present data demonstrated (Figure 2) that women run marathon at a more constant pace compared to men. This finding is in accordance with Deaner, Carter et al. (2015) who concluded that women were generally more effective compared to men in their marathon pacing. Furthermore, despite only analysing elite-level athletes, Hanley (2016) also found strong evidence that women were better at evenly pacing marathons than men: women ran the second half of the race closer to the pace of the first half. There are several hypothesis (Deaner, Lowen, Rogers, \& Saksa, 2015; Hanley, 2016; March, et al., 2011) aiming to explain sex differences in marathon pacing. The first states that the sex difference in marathon pacing is caused by the men's greater sensibility to muscle glycogen depletion (March, et al., 2011; Tarnopolsky, 2008), a major contributor to dramatic decline in marathon pace towards the end of the race (Coyle, 2007). There is a considerable amount of facts indicating that at a given intensity of endurance exercise, women take advantage of low glycogen conditions (Thibault, et al., 2010) compared to men. Secondly, during endurance exercise, women generally have a lower respiratory exchange ratio indicating they utilize proportionately more fat and less carbohydrate at a given intensity of exercise (Lamont, 2005). Thirdly, an alternative hypothesis proposed by Allen and Dechow (2013), is that the sex difference in marathon pacing is due to sex differences in decisionmaking. Men may select more ambitious starting paces, which increases the risk of slowing down due 
to the fact that they are more oriented to time goals (Allen \& Dechow, 2013) and more prone to take risks (Deaner, Lowen, et al., 2015). Men may more likely adopt a risky pace, relative to their ability, which could increase their probability of slowing down during the second part of the race.

The present study showed that veterans ran at a more constant pace trough the marathon. When time group and sex were taken into account, veteran runners maintained a slightly more even pace throughout the maraton course compared to the non-veteran runners. It has been hypothesised that older runners' pacing is more even because of their greater experience (March, et al., 2011), therefore knowing better their own abilities and being more skillful in distributing pace over the marathon distance (March, et al., 2011).

Limitations of the present study was the comparison of only the first and the second half of marathon and not including 5 or $10 \mathrm{~km}$ split times; also, the limited data available about the age groups. However, the strength of the study was including a high number of finishers with wide range of performance level compared to previously published studies (Haney \& Mercer, 2011; Hanley, 2016; March, et al., 2011).
In conclusion, irrespective of sex and age, faster finishers maintain a more constant velocity than the slower ones. Furthermore, women are generally more effective than men in choosing their marathon pacing. The causes of the sex difference in pacing are not clear cut, however, they likely involve the interplay of physiological and psychological factors.

As the number of recreational runners participating in the marathons has increased over the years, the results of the present study would be of great interest to high-level marathon runners and their coaches as well as to recreational runners aiming to finish their first marathon successfully. The vast majority of recreational athletes start their marathon competition relatively fast compared to the second half and thus decline their speed towards the end of the race. The results of the present study showed that finishers who had the smallest difference between the first and second half of the marathon race, performed better compared to the finishers with the biggest difference between the first and second half of the marathon. Furthermore, female runners tended to choose a slightly more even pacing strategy compared to male runners.

\section{References}

Abbiss, C.R., \& Laursen, P.B. (2008). Describing and understanding pacing strategies during athletic competition. Sports Medicine, 38(3), 239-252.

Abbott World Marathon Majors. (2016). AWMM. Retrieved March 3, 2016, from https://www.worldmarathonmajors.com/

Allen, E.J., \& Dechow, P.M. (2013). The 'rationality'of the long distance runner: Prospect theory and the marathon. Rochester, NY: Social Science Research Network.

Angus, S.D. (2014). Did recent world record marathon runners employ optimal pacing strategies? Journal of Sports Sciences, 32(1), 31-45.

Association of Road Racing Statisticians. (2016). ARRS. Retrieved March 6, 2016, from http://arrs.net/

Cohen, J. (1988). Statistical power analysis for the behavioural sciences. Hillside, NJ: Lawrence Earlbaum Associates.

Coyle, E.F. (2007). Physiological regulation of marathon performance. Sports Medicine, 37(4-5), 306-311.

Deaner, R.O., Carter, R.E., Joyner, M.J., \& Hunter, S.K. (2015). Men are more likely than women to slow in the marathon. Medicine and Science in Sports and Exercise, 47(3), 607-616.

Deaner, R.O., Lowen, A., Rogers, W., \& Saksa, E. (2015). Does the sex difference in competitiveness decrease in selective sub-populations? A test with intercollegiate distance runners. PeerJ, 3, e884.

de Koning, J.J., Bobbert, M.F., \& Foster,C. (1999). Determination of optimal pacing strategy in track cycling with an energy flow model. Journal of Science and Medicine in Sport, 2(3), 266-277.

Ely, M.R., Martin, D.E., Cheuvront, S.N., \& Montain, S.J. (2008). Effect of ambient temperature on marathon pacing is dependent on runner ability. Medicine and Science in Sports and Exercise, 40(9), 1675-1680.

Foster, C., de Koning, J.J., Bischel, S., Casolino, E., Malterer, K., O’Brien, K., Rodriguez-Marroyo, J., Splinter, A., Thiel, C., \& Van Tunen, J. (2012). Pacing strategies for endurance performance. In I. Mujika (Ed.), Endurance training, science and practice (pp 89-98). Vitoria-Gasteiz: Victoria-Gasteiz Press.

Foster, C., de Koning, J.J., Hettinga, F., Lampen, J., Dodge, C., Bobbert, M., \& Porcari, J.P. (2004). Effect of competitive distance on energy expenditure during simulated competition. International Journal of Sports Medicine, 25(3), 198-204.

Haney Jr., T.A., \& Mercer, J.A. (2011). A description of variability of pacing in marathon distance running. International Journal of Exercise Science, 4(2), 133-140. 
Hanley, B. (2016). Pacing, packing and sex-based differences in Olympic and IAAF World Championship marathons. Journal of Sports Sciences, 34(17), 1675-1681.

Hopkins, W.G., \& Hewson, D.J. (2001). Variability of competitive performance of distance runners. Medicine and Science in Sports and Exercise, 33(9), 1588-1592.

IAAF Competition Rules. (2014, 2015). Retrieved March 10, 2016, from https://www.iaaf.org/about-iaaf/documents/ rules-regulations

Lamont, L.S. (2005). Gender differences in amino acid use during endurance exercise. Nutrition Reviews, 63(12), 419-422.

March, D.S., Vanderburgh, P.M., Titlebaum, P.J., \& Hoops, M.L. (2011). Age, sex, and finish time as determinants of pacing in the marathon. Journal of Strength and Conditioning Research, 25(2), 386-391.

Marino, F.E. (2004). Anticipatory regulation and avoidance of catastrophe during exercise-induced hyperthermia. Comparative Biochemistry and Physiology Part B: Biochemistry and Molecular Biology, 139(4), 561-569.

Roelands, B., de Koning, J., Foster, C., Hettinga, F., \& Meeusen, R. (2013). Neurophysiological determinants of theoretical concepts and mechanisms involved in pacing. Sports Medicine, 43(5), 301-311.

Sandals, L.E., Wood, D.M., Draper, S.B., \& James, D.V.B. (2006). Influence of pacing strategy on oxygen uptake during treadmill middle-distance running. International Journal of Sports Medicine, 27(1), 37-42.

Santos-Concejero, J., Tam, N., Granados, C., Irazusta, J., Bidaurrazaga-Letona, I., Zabala-Lili, J., \& Gil, S.M. (2014). Interaction effects of stride angle and strike pattern on running economy. International Journal of Sports Medicine, 35(13), 1118-1123.

Santos-Lozano, A., Collado, P.S., Foster, C., Lucia, A., \& Garatachea, N. (2014). Influence of sex and level on marathon pacing strategy. Insights from the New York City race. International Journal of Sports Medicine, 35(11), 933-938.

Tarnopolsky, M.A. (2008). Sex differences in exercise metabolism and the role of 17-beta estradiol. Medicine and Science in Sports and Exercise, 40(4), 648-654.

Thibault, V., Guillaume, M., Berthelot, G., El Helou, N., Schaal, K., Quinquis, L., Nassif, H., Tafflet, M., Escolano, S., Hermine, O., \& Toussaint, J.F. (2010). Women and men in sport performance: The gender gap has not evolved since 1983. Journal of Sports Science and Medicine, 9(2), 214-223.

Thompson, K.G., MacLaren, D.P., Lees, A., \& Atkinson, G. (2004). The effects of changing pace on metabolism and stroke characteristics during high-speed breaststroke swimming. Journal of Sports Sciences, 22(2), 149-157.

Thompson, K., MacLaren, D., Lees, A., \& Atkinson, G. (2003). The effect of even, positive and negative pacing on metabolic, kinematic and temporal variables during breaststroke swimming. European Journal of Applied Physiology, 88(4-5), 438-443.

Submitted: February 27, 2017

Accepted: April 20, 2018

Published Online First: August 31, 2018

Correspondence to:

Martin Mooses

Institute of Sport Sciences and Physiotherapy

University of Tartu

Jakobi 5-209, 51014 Tartu, Estonia

Phone: +372 53463066

E-mail: Martin.Mooses@ut.ee. 